\title{
Téoros
}

Revue de recherche en tourisme

\section{Inventaire des impacts des activités récréatives en milieu forestier protégé}

\author{
Le cas du parc de la forêt ancienne du mont Wright
}

\section{Véronique Audet, Vincent Seigner et Julie Molard}

Volume 25, numéro 3, automne 2006

Tourisme et forêt, visions d’une gestion intégrée

URI : https://id.erudit.org/iderudit/1071020ar

DOI : https://doi.org/10.7202/1071020ar

Aller au sommaire du numéro

Éditeur(s)

Université du Québec à Montréal

ISSN

0712-8657 (imprimé)

1923-2705 (numérique)

Découvrir la revue

Citer cet article

Audet, V., Seigner, V. \& Molard, J. (2006). Inventaire des impacts des activités récréatives en milieu forestier protégé : le cas du parc de la forêt ancienne du mont Wright. Téoros, 25(3), 52-58. https://doi.org/10.7202/1071020ar 


\section{Inventaire des impacts des activités récréatives en milieu forestier protégé Le cas du parc de la forêt ancienne du mont Wright ${ }^{1}$}

\section{Véronique Audet, Vincent Seigner et Julie Molard}

Dans l'optique d'un développement récréotouristique durable, particulièrement dans le cas des milieux forestiers protégés, il est essentiel de mettre en place des moyens et des modes de gestion efficaces pour s'assurer du maintien de l'intégrité écologique de ces espaces. Comme le mentionne le rapport de la Commission d'étude sur la gestion de la forêt publique québécoise, le réseau d'aires protégées québécois devra être bonifié. Le développement touristique dans ces milieux protégés pourra être un aspect de développement économique à valoriser, mais il faudra mettre en œuvre des moyens pour gérer les activités récréatives pratiquées afin de s'assurer du maintien de l'intégrité écologique.

À cet effet et dans un souci constant de préservation du milieu naturel, l'Association forestière Québec métropolitain (AFQM), gestionnaire de la mise en valeur et de la sensibilisation au parc municipal de la forêt ancienne du mont Wright, a élaboré une étude de suivi de l'intégrité écologique de ce parc. En effet, l'achalandage de plus en plus important n'est pas sans dommage sur ce milieu naturel. Les activités permises sur le site sont la randonnée pédestre et en raquettes ainsi que la pratique de l'escalade de parois et de blocs. L'objectif de cette étude visait à constituer un état des lieux en regard des impacts liés à la fréquentation par le public et à permettre le suivi, à long terme, de ces mêmes impacts. Pour répondre à cet objectif, l'étude a été divisée en deux grandes parties. La première partie consistait à mener une enquête portant sur les attitudes et les habitudes des usagers du mont Wright afin de mieux connaître le public qui fréquente le parc et la deuxième partie consistait à effectuer des relevés sur le terrain afin de qualifier et de quantifier les impacts de la fréquentation publique.

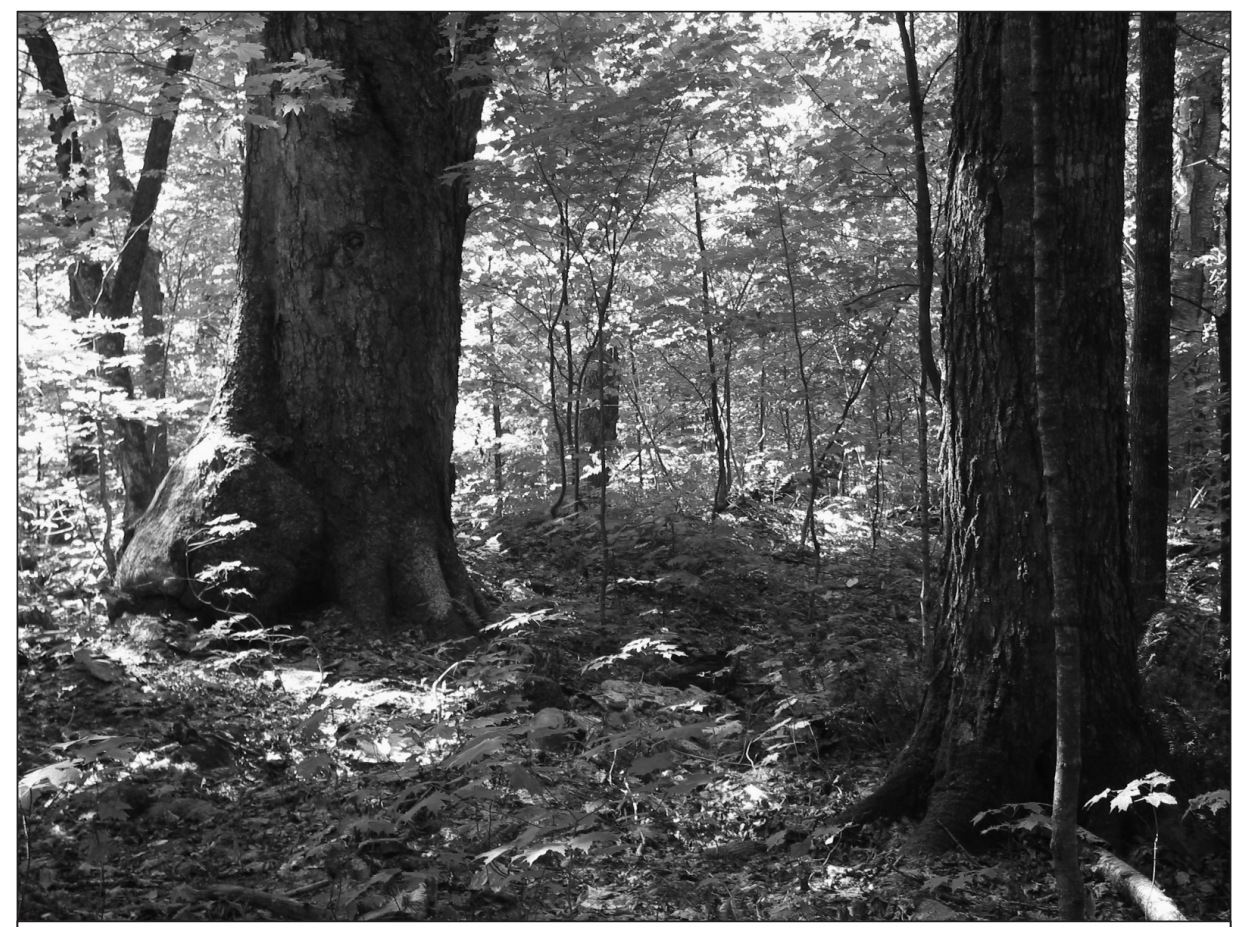

Érablière à bouleau jaune et hêtre ancienne.

Photo: Anick Lapointe, AFQM

Tout comme son nom l'indique, le parc de la forêt ancienne du mont Wright, d'une superficie de 187 hectares, est constitué de forêts anciennes. Les peuplements anciens identifiés sur le site sont une érablière à bouleau jaune et hêtre ainsi qu'une bétulaie jaune à sapin, toutes deux âgées de plus de 300 ans. Ces peuplements ont été reconnus comme écosystèmes forestiers exceptionnels (EFE) par le ministère des Ressources naturelles, de la Faune et des Parcs en 1997, mais cette reconnaissance ne lui confère aucune protection légale étant donné que le parc ne se trouve pas en forêt publique. Cependant, l'AFQM et la municipalité ont la volonté incontestable de protéger ce site unique et la présente étude constitue un outil majeur pour le maintien de son intégrité écologique.

\section{Méthodologie}

\section{Enquête sur les attitudes et les habitudes des usagers} du mont Wright

Le public qui fréquente le parc de la forêt ancienne du mont Wright est très peu connu puisque que ce parc n'est officiellement ouvert que depuis 2002. Afin de bien gérer la fréquentation du parc, il est indispensable de bien connaître les usagers. C'est pourquoi une enquête concernant le profil, les attitudes et les habitudes des usagers a été élaborée.

L'enquête a été réalisée sous la forme d'un questionnaire composé majoritairement de questions fermées à choix multiples pour réduire le temps et faciliter le traitement des 
données. Le mode de diffusion et de récolte de ce questionnaire s'est fait sur le terrain. Le questionnaire était anonyme, ce qui permet d'obtenir un maximum de franchise de la part des répondants.

Étant donné que la fréquentation du parc était inconnue, il était difficile de définir un échantillonnage suffisant pour être statistiquement fiable. Néanmoins, une limite minimale de 100 formulaires complétés a été fixée afin d'obtenir un échantillon relativement important.

\section{Suivi de l'intégrité écologique}

Cette partie de l'étude consistait à effectuer des relevés sur le terrain dans le but de qualifier et de quantifier finement les impacts de la fréquentation publique liés à la randonnée pédestre et à la pratique de l'escalade, particulièrement à l'escalade de blocs. Pour y parvenir, des placettes de sentiers, des placettes de blocs ont été utilisées et des relevés d'indicateurs semi-quantitatifs ont été effectués. La méthodologie s'appuie sur les travaux de Jeffrey Marion (1995 : 763-771), de David Cole et al. (1997) et sur une revue de la littérature de Yu-Fai Leung et Jeffrey Marion (2000 : 23-35). Les indicateurs ont, quant à eux, été choisis d'après les travaux d'Alan Watson et David Cole (1992: 65-84), de Siobhan Jackson et Doug Leavers (2000) et d'après un document de travail relatif au programme de suivi de l'intégrité écologique (PSIE) de Parcs Québec (2005).

\section{Placettes de sentiers}

Douze placettes de sentiers ont été utilisées, soit une tous les 350 mètres. Cette fraction d'échantillonnage est inférieure à celle fixée par Leung et Marion (1999: 167-179), leurs résultats préconisant un échantillonnage d'un point tous les 500 mètres. Ces placettes avaient pour principal objectif d'évaluer l'impact de la randonnée pédestre sur et en bordure des sentiers qui ne résultent pas de réaménagement d'anciens sentiers. Chacune des placettes a été découpée en trois portions (voir figure 1) :

- la zone S (pour sentier) correspond à la largeur du sentier sur une distance de 10 mètres ;

- la zone B (pour bordure) est composée de bandes de 2 mètres de largeur de chaque côté du sentier ;

- la zone P (pour peuplement) correspond à une bande de 5 mètres de chaque côté de la zone B.

\section{Figure 1}

Placette de sentier

$x$ mètres +14 mètres

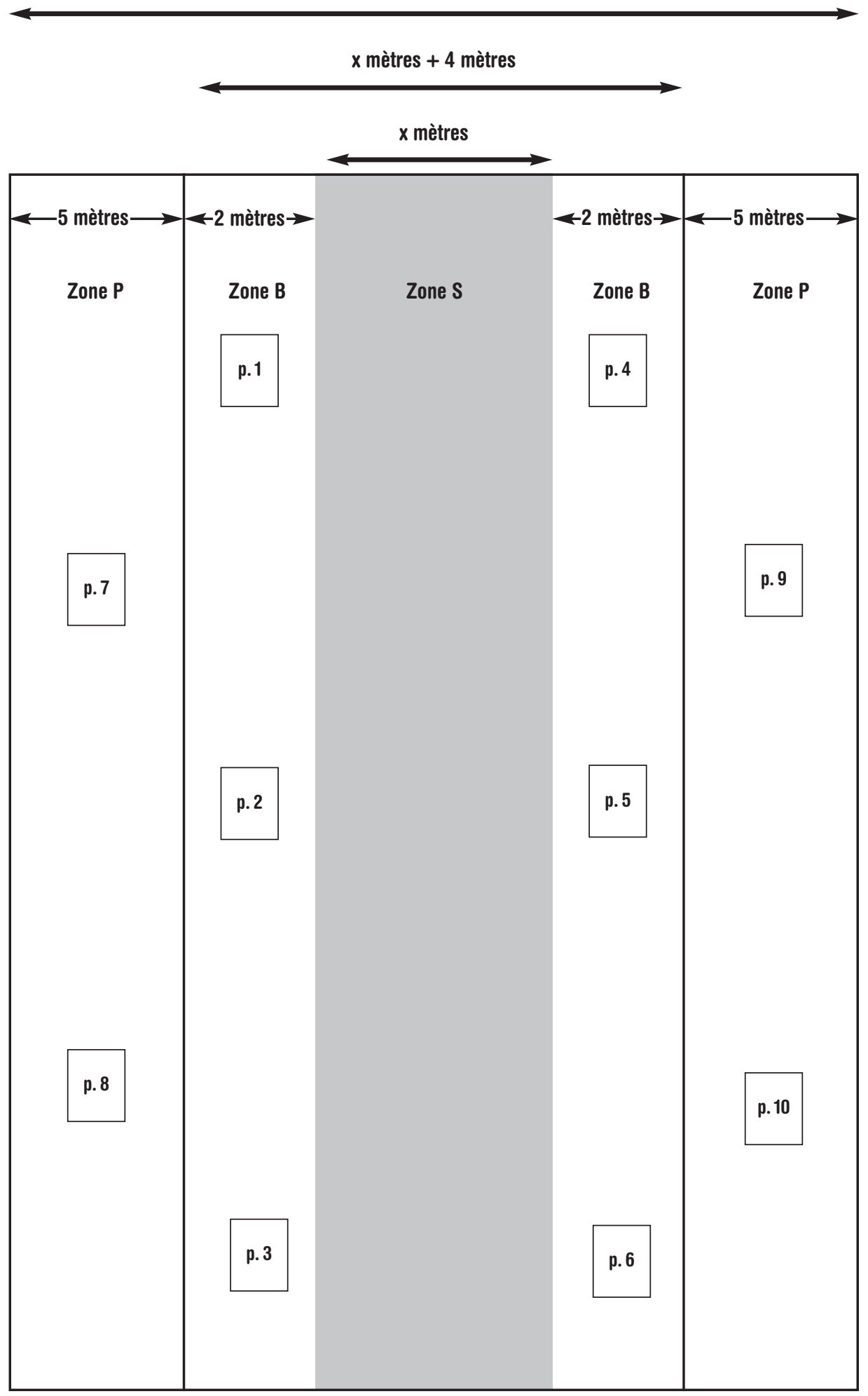

Source : Figure à partir des données de recherche des auteurs. 
La récolte des données concerne les indicateurs suivants : l'exposition des racines, la largeur des sentiers, le pourcentage de végétation et de sol mis à nu en bordure des sentiers ainsi que la présence de gravier 0,75 . L'exposition des racines a été relevée sur les zones $\mathrm{S}$ et $\mathrm{B}$. Cette exposition a été qualifiée selon trois catégories, soit : "aucune " lorsqu'il n'y a pas de racine apparente, "moyenne " quand les racines sont peu visibles et que la majorité d'entre elles ne sont pas exposées à plus de la moitié et "forte " lorsqu'il y a beaucoup de racines observables et qu'elles ressortent fortement du sol.

Pour ce qui est de la largeur des sentiers, les relevés ont été effectués uniquement sur la zone S. La largeur du sentier était relevée trois fois par placette afin d'établir une moyenne, soit un relevé à chaque extrémité de placette, plus un au centre.

Le pourcentage de sol mis à nu et le pourcentage de végétation ont été mesurés en zones $\mathrm{B}$ et $\mathrm{P}$. Le pourcentage de sol mis à nu est exprimé par des tranches de pourcentage à la dizaine (ex: 10 \%, 20\%, $30 \%$ ), représentant la surface occupée par le sol mis à nu. Les mesures ont été effectuées à l'aide d'un cadre de bois d'une surface de un mètre carré. Dix relevés de ce type ont été effectués par placette, six en zone B et quatre en zone $\mathrm{P}$.

\section{Placettes de blocs}

Les placettes de blocs, au nombre de 40, ont été localisées sur tous les blocs identifiés sur le topo du secteur droit du parc (Dubuc, Gerrits et al.). Elles étaient formées des blocs et de la surface au sol qui est touchée par le piétinement. Leur principal objectif était de mettre en évidence l'impact de la pratique de l'escalade de blocs sur les blocs et sur la végétation à proximité.

La récolte des données concernait les indicateurs de végétalisation et de piétinement. Ces observations de l'indicateur de végétalisation se classaient en quatre catégories, soit : "aucune perturbation ", correspondant à l'état normal du bloc sans aucune perturbation causée par des actions anthropiques ; "présence de perturbations minimes ", correspondant à un bloc qui n'a pas connu de dégradation majeure, la végétation y étant encore re- lativement bien présente; "présence de perturbations importantes ", correspondant à un bloc qui a subi une forte perte de végétation; et "absence de végétation ", regroupant les blocs qui sont totalement ou en majeure partie dépourvus de végétation.

Pour ce qui est de l'indicateur de piétinement, il consistait à mesurer la largeur de la bande piétinée au pied des blocs. Dix opérations de ce type, réparties équitablement autour du bloc étudié, ont été effectuées sur chaque placette. Les dix données ont permis d'établir la largeur moyenne de végétation fortement touchée par le piétinement à proximité des blocs.

\section{Relevés d'indicateurs semi-quantitatifs}

Les indicateurs semi-quantitatifs ont été évalués par un cheminement sur tout le réseau de sentiers primaires et secondaires du parc. Les indicateurs concernaient le nombre de raccourcis, l'état du réseau de sentiers secondaires et l'état de leur minéralisation, le dérangement du drainage et le nombre d'actes de vandalisme.

L'indicateur relié au nombre de raccourcis était relevé en effectuant un cheminement sur tous les sentiers primaires. Pour ce qui est de l'état du réseau de sentiers secondaires, un relevé GPS a permis d'estimer la localisation et la distance couverte par ce réseau de sentiers informels. Sur ces mêmes sentiers, l'état de minéralisation du sol a été qualifié grâce à des observations visuelles de la végétation et de l'humus classifié en trois catégories. La catégorie 1 était choisie lorsque la végétation était encore présente sur le sentier informel et les horizons humifères n'étaient pas trop endommagés, la catégorie 2 lorsque la végétation était absente et/ou les horizons humifères étaient endommagés et la catégorie 3 lorsque la végétation et les horizons humifères étaient absents. Ces observations étaient répétées à une fréquence de un point de sondage tous les 100 mètres.

Quant au dérangement du drainage, il s'agissait d'une observation visuelle qui consistait à estimer l'état du drainage des aménagements, dont les fossés, les drains et les rigoles d'évacuation d'eau. L'état de ces aménagements a été qualifié selon trois catégories, soit « acceptable " lorsqu'il permettait un bon écoulement de l'eau et qu'il était en bon état, "passable » si l'écoulement de l'eau était gêné par un manque d'entretien ou le mauvais état de l'aménagement et "critique » quand le passage de l'eau était obstrué.

Le nombre d'actes de vandalisme est un indicateur qui a été est relevé en cheminant dans tous les sentiers primaires.

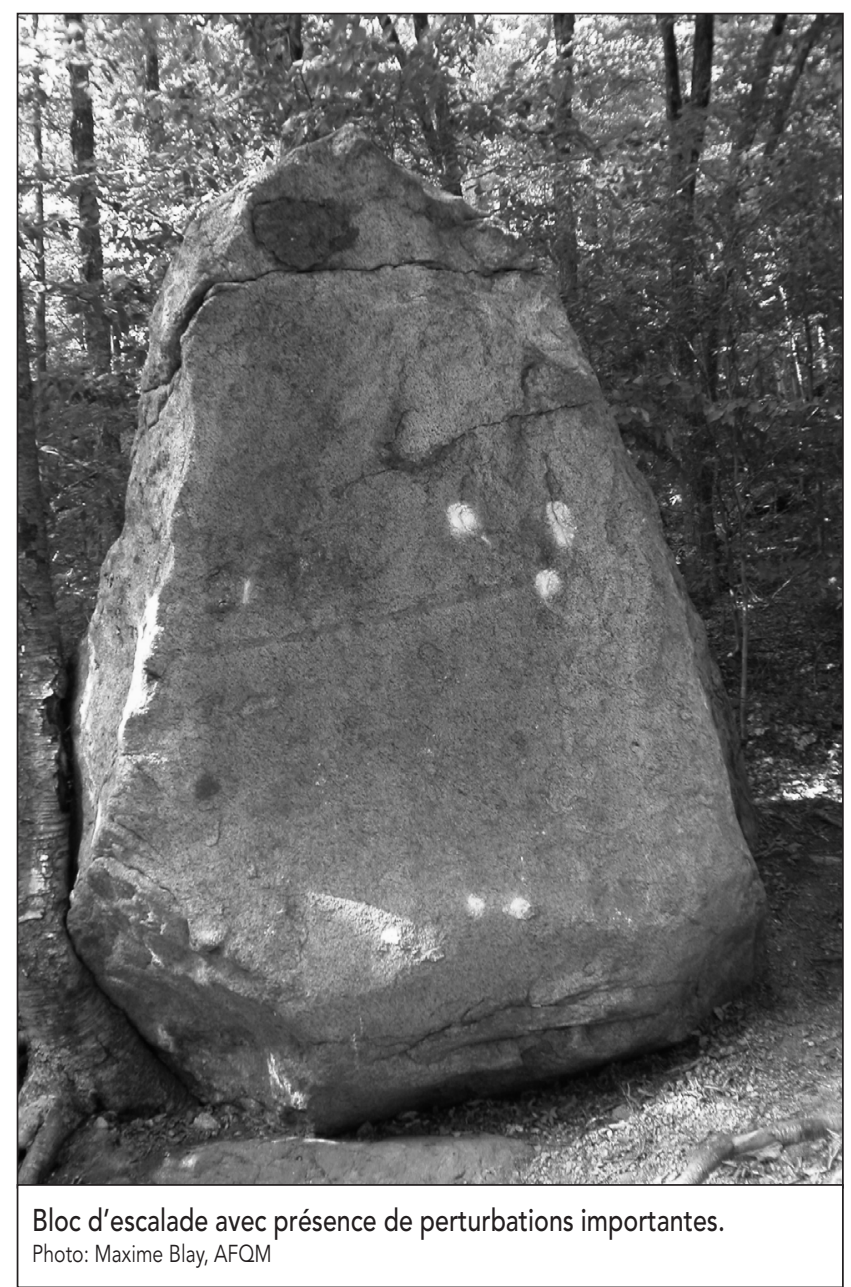




\section{Résultats}

\section{Enquête sur les attitudes et les habitudes des usagers du mont Wright}

L'enquête portant sur les attitudes et les habitudes des usagers du parc a interpellé 124 répondants, dépassant ainsi l'objectif fixé de 100 répondants. Selon les données recueillies concernant le profil des usagers, le parc est fréquenté tant par des grimpeurs (55\%) que par des randonneurs (45\%). On remarque également que la majorité des usagers du parc (60\%), grimpeurs et randonneurs confondus, le fréquentent depuis peu de temps. II est intéressant de noter par ailleurs qu'environ $10 \%$ des usagers du parc fréquentaient le parc avant son ouverture officielle en 2002. Pour les grimpeurs, l'attrait principal de ce parc est l'escalade (55\%), bien que $19 \%$ d'entre eux apprécient la randonnée dans le parc. L'escalade de paroi est le type d'escalade le plus prisé et le plus pratiqué avec $65 \%$ des grimpeurs sondés qui la pratiquent, contre $35 \%$ qui sont adeptes du bloc.

Pour ce qui est de l'impact de la sensibilisation sur le public, on constate que le règlement municipal appliqué au parc semble être connu par moins de la moitié des usagers, soit $41 \%$ des grimpeurs et $44 \%$ des randonneurs. Le zonage du parc est quant à lui pratiquement inconnu du public. En effet, $68 \%$ des grimpeurs et $78 \%$ des randonneurs ne connaissent pas le zonage du parc. II est aussi à noter que $76 \%$ des grimpeurs et $78 \%$ des randonneurs affirment ne pas savoir ce qu'est un écosystème forestier exceptionnel, qui constitue pourtant l'élément majeur de la conservation de ce milieu naturel.

L'analyse des attitudes des usagers montre que les sentiers formels semblent être respectés par la majorité des répondants. En effet, $66 \%$ des randonneurs et $67 \%$ des grimpeurs affirment ne jamais utiliser de sentiers informels. II y a donc environ un tiers des usagers qui disent utiliser des sentiers informels de temps en temps. Seulement $10 \%$ des grimpeurs recherchent de nouvelles parois ou de nouveaux blocs dans le parc. Lorsque les visiteurs croisent d'autres personnes sur les sentiers, la majorité d'entre eux a comme principal réflexe de se placer sur un bord du sentier et de continuer à marcher. Pour ce qui est des actions interdites par le règlement du parc, notons que les chiens en liberté, qui concernent $3 \%$ des grimpeurs et $15 \%$ des randonneurs, et le camping, qui concerne $1 \%$ des grimpeurs et $3 \%$ des randonneurs, sont les infractions les plus fréquentes.

Quant aux habitudes des usagers, les grimpeurs fréquentent le parc principalement l'été dans une proportion de $53 \%$ et les randonneurs le fréquentent toute l'année dans une proportion de $31 \%$. La période la moins fréquentée est donc l'hiver, avec 1\% des grimpeurs et $35 \%$ des randonneurs. Les grimpeurs, comme les randonneurs, effectuent leurs visites en groupe de moins de 4 personnes, soit $42 \%$ des grimpeurs et $77 \%$ des randonneurs. Les grimpeurs sont ceux qui ont tendance à venir en plus grand groupe : $26 \%$ d'entre eux viennent en groupe de plus de 8 personnes. En matière d'escalade, les grimpeurs considèrent l'utilisation du carbonate de magnésium comme préférable à hauteur de $49 \%$ et d'optionnelle à hauteur de $19 \%$. Le nettoyage des parois semble être effectué, pour la plupart (53\%), lorsque cela est nécessaire. Notons cependant que $1 \%$ des grimpeurs nettoient la paroi à chaque fois. Les besoins personnels des grimpeurs, qui sont généralement présents des journées entières sur le site, sont effectués à $77 \%$ aux toilettes chimiques situées sur le stationnement et à $19 \%$ n'importe où en forêt.

\section{Suivi de l'intégrité écologique}

\section{Placettes de sentiers}

Pour ce qui est de l'exposition racinaire en zone $\mathrm{S}$, il y en a majoritairement "aucune " (42\%). Cependant, plus de la moitié des placettes ont une exposition des racines qualifiée de "moyenne» (33\%) ou de "forte » $(25 \%)$. L'exposition racinaire en zone $\mathrm{B}$ est majoritairement qualifiée de «moyenne », mais il est important de noter qu'aucune placette a une exposition des racines qualifiée de « forte » dans la zone $\mathrm{B}$. La largeur moyenne des sentiers varie de 87 à 177 centimètres. La majorité des placettes a une largeur moyenne variant de 100 à 140 centimètres. Pour ce qui est de la végétation, il y a plus de sol mis à nu que de végétation en zone $B$. En zone $P$, les pourcentages considérés n'ont pas de tendance dominante marquée.
Quant à la présence de gravier 0,75, cet indicateur concerne uniquement certaines placettes qui sont les seules à se retrouver sur une portion de sentier qui a été gravelée lors de leur construction. Les autres placettes sont sur des portions de sentier recouvertes d'un substrat naturel. Dans la majorité des cas, le gravier est encore relativement présent (de 75 à 100 \%) sur le sentier et aucune placette n'a un recouvrement en gravier 0,75 inférieur à $50 \%$. Cependant, certaines placettes connaissent une perte de matériaux.

\section{Placettes de blocs}

Du côté de l'indicateur de végétalisation, la majorité des blocs inventoriés présentent des perturbations, soit minimes (50\%), soit importantes (44\%). Notons que $47 \%$ des blocs inventoriés sont officiellement ouverts au public. II est intéressant de constater qu'aucun bloc ne présente une absence totale de végétation.

Le piétinement autour des blocs va de 0 à 510 centimètres, avec une moyenne de 84 centimètres. Dans la plupart des cas, les blocs présentant des perturbations minimes ont une largeur piétinée faible alors que les blocs présentant des perturbations importantes ont une largeur piétinée assez importante.

\section{Relevés d'indicateurs semi-quantitatifs}

II y a 30 raccourcis sur le réseau de sentiers du parc qui totalisent 6 kilomètres. Vingt-cinq d'entre eux se concentrent principalement sur le sentier le plus utilisé par les usagers du parc. Les actes de vandalisme sont majoritairement localisés au sommet et les feux à ciel ouvert sont les plus communs.

Le réseau de sentiers secondaires s'étend sur une distance de 6750 mètres. Ces sentiers se concentrent principalement autour des blocs dans la forêt ancienne, au-dessus de la paroi et entre les abris sous roche et le sommet. Une grande partie de ces sentiers informels est balisée avec des fanions accrochés aux branches des arbres. Pour ce qui est de l'état de minéralisation, $44 \%$ des points de sondage ont une végétation encore présente, mais $31 \%$ de ceux-ci laissent paraître une absence totale de végétation et des horizons humifères. 
Sur le plan du drainage, il a été possible de constater que $59 \%$ des drains sont qualifiés de "passables", ce qui signifie que l'écoulement de l'eau est gêné par un manque d'entretien ou le mauvais état de l'aménagement. Les drains qui ont le mieux résisté sont les gros drains fermés. Les drains ouverts, dont $60 \%$ sont qualifiés de "passables ", ont tendance à accumuler une quantité importante de feuilles mortes et de débris ligneux, mais se comportent tout de même relativement bien. Les drains fermés, dont $86 \%$ sont qualifiés de "passables " à " critiques ", se bouchent très facilement. Notons également qu'ils se déterrent fréquemment et que $29 \%$ d'entre eux sont mis à nu sur $75 \%$ de leur longueur. Le principal problème observé est un amas d'obstacles qui limite ou empêche l'écoulement de l'eau. Certains drains sont tellement bouchés que l'écoulement de l'eau suit un itinéraire différent de celui qui est souhaité, entraînant ainsi des pertes de sol. Les enrochements effectués en soutien s'avèrent efficaces dans la majorité des cas. Cependant, certains d'entre eux favorisent les amas d'obstacles qui freinent l'écoulement de l'eau. II est important de signaler qu'aucun entretien du réseau de drainage n'a été effectué depuis son installation en 2002.

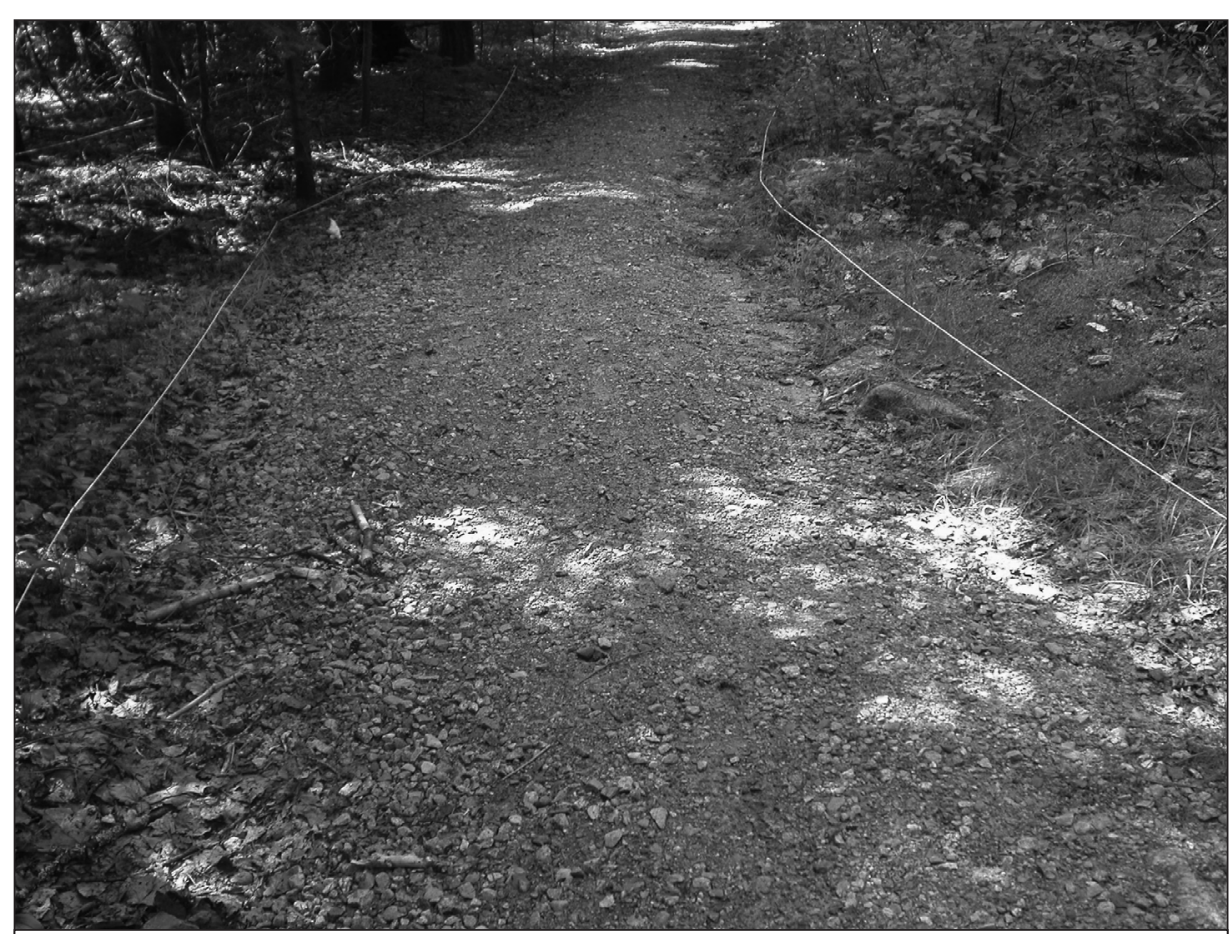

Sentier gravelé avec aucune exposition racinaire.

Photo: Vincent Seigner/AFQM

\section{Discussion}

\section{Enquête sur les attitudes et les habitudes des usagers du mont Wright}

Selon le profil des usagers, les actions menées sur le parc ne devraient pas prioriser une activité plutôt que l'autre, car les deux activités autorisées sur le parc sont pratiquées à part égale. Le gestionnaire devrait permettre la poursuite et le maintien de ces deux activités en évitant les conflits d'usage, la forêt. On remarque que 10 \% des usagers du parc le fréquentaient avant son ouverture, ce qui explique certaines habitudes, pas nécessairement souhaitables, qui ont été prises antérieurement.

Selon l'enquête, la sensibilisation effectuée dans ce parc ne semble pas être efficace pour tous les usagers. Les panneaux d'interprétation ne semblent pas être lus. Si les panneaux d'interprétation et les règlements étaient lus, la connaissance des usagers relative à l'information véhiculée serait meilleure qu'elle ne l'est présentement. II est donc nécessaire d'accentuer les actions de sensibilisation dans le but de conscientiser les usagers du parc à la fragilité de ce milieu. tout en maintenant l'intégrité écologique de
Il est également important de dissuader les usagers qui empruntent les sentiers informels ainsi que les grimpeurs qui recherchent de nouvelles parois ou de nouveaux blocs, afin de diminuer les impacts sur le milieu naturel. Une concertation des différents intervenants permettrait la recherche de solutions en ce sens.

Lorsque les usagers du parc se croisent sur les sentiers, leur premier réflexe est de se placer sur un côté du sentier et de continuer à marcher. Cette attitude a des effets de piétinement sur les bordures de sentiers. Conséquemment, lors des campagnes de sensibilisation, il est nécessaire d'expliquer aux personnes qui fréquentent le parc l'importance de s'arrêter sur un côté du sentier pour laisser passer l'autre groupe de personnes, limitant ainsi l'impact sur la bordure du sentier. II est également important de sensibiliser l'ensemble des usagers à l'importance de rapporter leurs déchets avec eux et de les déposer aux endroits prévus, car, malgré qu'ils prétendent le faire presque unanimement, des déchets sont encore observables sur le territoire du parc.

L'action interdite la plus fréquemment avouée est la promenade de chien sans laisse. C'est une problématique réelle dans le parc, car plusieurs propriétaires de chien s'approprient ce droit. Le parc de la forêt ancienne du mont Wright, contrairement aux parcs nationaux du Québec, accepte les animaux domestiques, ce qui a pour conséquence d'attirer bon nombre de propriétaires de chien.

Le fait que les visites au parc s'effectuent majoritairement en groupe de moins de 4 personnes est un point positif. Selon Dana Elle (2000), il est préférable de composer des groupes de petite taille, inférieurs à 8 personnes. Alpine Adventures (2004) conseille même de ne pas dépasser 3 à 4 personnes.

L'utilisation du carbonate de magnésium et le brossage des parois par les adeptes de l'escalade sont des sujets très controversés chez les grimpeurs. II serait intéressant de limiter l'utilisation du carbonate de magnésium et du brossage afin de réduire l'impact sur les parois et les blocs. Par ailleurs, à la suite de l'enquête et de commentaires recueillis, l'installation d'une toilette sèche à proximité de la grande paroi d'escalade pourrait réduire le nombre de grimpeurs qui vont effectuer leurs besoins personnels n'importe où en forêt (19\%). 


\section{Suivi de l'intégrité écologique}

\section{Placettes de sentiers}

Le fait que la zone S n'ait pratiquement aucune exposition racinaire s'explique par la présence du gravier 0,75 sur certaines placettes. En effet, sur les cinq placettes qui ont été gravelées, quatre n'ont aucune exposition racinaire. En revanche, il n'y a qu'une seule placette sur les sept qui ne sont pas recouvertes de gravier qui n'a aucune exposition des racines. Ces résultats mettent donc en évidence l'effet positif du gravier 0,75 . II protège le système racinaire des arbres situés en bordure de sentier et limite les effets de tassement du substrat lié au passage répété des marcheurs. Le gravier 0,75 reste la plupart du temps là où il a été posé. Les zones qui présentent des départs de matériaux sont des portions de sentiers où la pente est assez forte. Le passage répété de randonneurs et l'écoulement de l'eau lors des fortes pluies sont les principaux facteurs responsables de ces départs de matériaux. En zone B, l'exposition des racines est majoritairement qualifiée de moyenne, ce qui met en évidence le fait que les marcheurs empiètent sur la bordure des sentiers. II est important de mettre ces résultats en corrélation avec la largeur des sentiers, car le piétinement de la bordure des sentiers conduit inévitablement à leur élargissement. La largeur des sentiers est relativement constante. Elle est majoritairement comprise de 100 à 140 centimètres, des valeurs relativement acceptables pour l'utilisation des sentiers dans le parc. II est donc important de veiller à maintenir la largeur des sentiers dans cette fourchette. Rappelons que la création d'un sentier a des impacts sur le milieu naturel et que moins les sentiers sont larges, plus les impacts sur le milieu naturel sont réduits.

Les données sur le pourcentage de sol mis à nu ne représentent pas nécessairement le piétinement des usagers du parc, mais plutôt la proportion de sol qui n'est pas recouverte de végétation. En revanche, par comparaison d'inventaire, ce pourcentage de sol mis à nu pourra éventuellement mettre en évidence un impact de la fréquentation publique sur le recouvrement en végétation. L'ouverture des sentiers ne semble pas avoir eu d'impact sur la végétation par la présence, par exemple, d'une plus grande quantité de lumière au sol. Les impacts observés semblent davantage être liés au piétinement. En effet, étant donné que le pourcentage de sol mis à nu est plus important en zone B qu'en zone $\mathrm{P}$, on peut supposer que le passage répété des randonneurs affecte le recouvrement de la végétation.

\section{Placettes de blocs}

Les blocs ouverts au public ont subi un impact sur la végétation qui est moins marqué que celui des blocs qui sont interdits. Cela peut s'expliquer de différentes façons. Tout d'abord, les blocs ouverts au public ont été officiellement déterminés depuis peu de temps et les grimpeurs ne sont pas tous informés de cette situation. Par ailleurs, les blocs fermés au public sont situés au milieu des peuplements forestiers, alors que les blocs ouverts au public sont situés en bordure de sentier. Ainsi, les blocs fermés au public sont moins exposés à la lumière et doivent recevoir moins de précipitations en raison du couvert forestier. Dans ces conditions, la végétation se développe moins vite sur les blocs qui sont sous couvert forestier que sur ceux qui sont situés en bordure de sentier. Ces hypothèses restent toutefois à être confirmées lors des prochains inventaires.

Le piétinement est étroitement lié à l'état de végétalisation des blocs. Ces deux facteurs permettent de déterminer quels sont les blocs qui sont grimpés. Le piétinement est inévitable, mais on peut éviter, par des mesures de gestion appropriées, qu'il atteigne une largeur maximale de 510 centimètres telle qu'observée lors de l'étude. II est intéressant de constater que la majorité des blocs ont une largeur piétinée maximale inférieure à 300 centimètres. Compte tenu de la largeur des crash pads ${ }^{2}$ et de la distance nécessaire pour " assurer ${ }^{3}$ " correctement le grimpeur, nous considérerons qu'une largeur piétinée maximale de 300 centimètres pourrait être tolérée. L'impact de l'escalade de blocs est donc relativement important. Ce qui pose le plus de problèmes, c'est qu'une majeure partie des impacts recensés sont concentrés sur les blocs fermés au public. Ces blocs sont souvent situés dans les zones de conservation, ce qui peut avoir un effet important sur le maintien de l'intégrité écologique de ces zones fragiles.

\section{Relevés d'indicateurs semi-quantitatifs}

Du côté du nombre de raccourcis observés, on peut remarquer que ce sont les sentiers où la topographie est la plus forte qui sont touchés par les problèmes de raccourcis. De plus, ces sentiers sont sinueux pour en limiter l'érosion. On peut donc en déduire que les usagers du parc utilisent ces raccourcis par négligence ou pour gagner du temps. II est important de sensibiliser les usagers aux conséquences importantes de l'utilisation de ces raccourcis, par exemple la perte de végétation et l'érosion.

Au chapitre des actes de vandalisme, ceux-ci sont concentrés principalement au sommet. Certaines personnes choisissent en effet le belvédère situé au sommet pour passer des soirées autour d'un feu et même pour camper. Notons toutefois que les aménagements (abris et bancs) installés au sommet sont relativement attrayants pour les personnes qui souhaitent faire ces activités.

Les sentiers secondaires posent, quant à eux, une difficulté importante. En effet, une grande partie des sentiers secondaires sont situés dans la zone de préservation en plein cœur de la forêt ancienne. Les grimpeurs de blocs sont, entre autres, les responsables de la formation de ces nombreux sentiers. Cependant, les grimpeurs ne sont pas les seuls usagers de ces sentiers informels; certains randonneurs préfèrent sortir des sentiers battus pour chercher l'isolement. II est donc nécessaire de sensibiliser tous les usagers aux impacts qu'ils ont sur le milieu naturel.

Par ailleurs, $44 \%$ des sentiers secondaires présentent de la végétation, ce qui signifie que le piétinement n'y est pas encore trop important. II s'agit d'un point positif, car ces sentiers seront en mesure de se refermer assez rapidement. Les autres sentiers secondaires auront cependant davantage de difficultés à se revégétaliser, compte tenu du fait que les impacts observés sur ceux-ci sont plus importants. Néanmoins, cette situation n'est pas irréversible. Par exemple, le sentier de l'Acropole situé dans le parc national des Hautes-Gorges-de-la-Rivière-Malbaie, qui connaissait les mêmes problèmes de sentiers secondaires, montre bien qu'il est possible de revégétaliser des sentiers indésirables. Pour cela, on a utilisé des amas de branchages afin d'empêcher l'accès et de créer un apport de matière organique. Ces mesures ont été renforcées par une signalisation adaptée. 
Les aménagements visant la gestion du drainage semblent être relativement endommagés. Néanmoins, les impacts relevés n'ont rien de catastrophique et sont tous facilement résorbables. De plus, l'absence d'entretien de ces aménagements depuis 2002 explique leur état. Cet état des lieux mène à un autre constat qui est, quant à lui, positif. La majorité des drains mis en place continuent à bien diriger l'écoulement de l'eau. On peut donc dire que les drains présents sur cette portion du sentier de la forêt ancienne jouent encore leur rôle primaire qui est la gestion de l'écoulement de l'eau. Cependant, d'un point de vue paysager, les drains fermés ne sont pas aussi efficaces et la mise à nu de ce type de drain met en évidence l'effet de piétinement. En outre, dans ce cas-ci, l'érosion a été accentuée par le manque d'entretien; l'excès d'eau qui coule au-dessus des drains quand ils sont bouchés augmente le processus d'érosion.

\section{Conclusion et recommandations}

Selon les données recueillies et les impacts observés dans cette étude, il est possible d'émettre certaines recommandations qui permettront une meilleure gestion des activités récréatives dans le parc. Ces recommandations ont pour but de contribuer à réduire les impacts afin de maintenir l'intégrité écologique du site et particulièrement des forêts anciennes. La principale recommandation consiste à poursuivre et à développer de nouvelles méthodes de sensibilisation des usagers en regard de la fragilité du site, de l'importance du respect de la réglementation et du zonage et à maintenir un lien étroit avec les organismes responsables du site d'escalade. Par la suite, afin de réduire les impacts de la randonnée pédestre, il est suggéré, en plus de la sensibilisation, d'améliorer le balisage et la signalisation des sentiers. L'entretien et le suivi des infrastructures de drainage est également un aspect primordial pour assurer un drainage efficace et un aménagement durable des sentiers. Finalement, afin de gérer efficacement les deux principales activités pratiquées dans le parc, il est recommandé d'élaborer un plan de gestion des activités récréatives de l'escalade et de la randonnée pédestre. Ces outils de gestion permettront de cibler précisément des actions à mettre en œuvre pour réduire les répercussions sur le milieu naturel et pour améliorer la qualité de l'accueil des usagers.
L'étude sur les attitudes et les habitudes des usagers du parc de la forêt ancienne du mont Wright nous a permis de mieux connaître les usagers de ce parc, ce qui s'avère un élément permettant de mieux cibler les interventions et particulièrement les modes de sensibilisation. Nous savions avant la réalisation de cette étude que la fréquentation du public avait un certain nombre d'impacts sur le milieu naturel. Cependant, la réalisation d'une étude comme celle-ci a permis de quantifier et de mettre en évidence des impacts dont nous avions moins conscience, par exemple l'étendue du réseau de sentiers secondaires.

La majorité des indicateurs relevés dans cette étude seront beaucoup plus significatifs lors de la prochaine mise en œuvre. En effet, aucun relevé n'avait été réalisé avant cette année et cette campagne d'inventaire constituait principalement un état des lieux. Cependant, plusieurs hypothèses, constats et recommandations ont pu être avancés. Lors du prochain inventaire, il sera possible d'effectuer des comparaisons qui permettront de confirmer ou d'infirmer les hypothèses mises de l'avant et de mesurer la réussite des mesures d'atténuation mises en place. Cette étude constitue donc un outil permettant de mieux gérer les activités récréatives pratiquées dans le parc et de minimiser les impacts afin de favoriser le maintien de l'intégrité écologique de ce milieu naturel protégé.

Véronique Audet est chargée de projets à l'Association forestière Québec métropolitain.

Vincent Seigner est technicien supérieur en management des ressources forestières et stagiaire responsable de l'étude.

Julie Molard est directrice générale à l'Association forestière Québec métropolitain.

\section{Notes}

1 L'étude a été réalisée grâce au soutien technique et scientifique de Louis Lefevbre, conseiller en loisir et récréation de plein air au ministère du Développement durable, de l'Environnement et des Parcs, de Jean Gagnon, biologiste spécialisé en végétation au même ministère, et de Louis Bélanger, ingénieur forestier de la Faculté de foresterie et de géomatique de l'Université Laval, professeur et chercheur.
2 Matelas de sécurité disposé au sol afin de protéger les grimpeurs en cas de chute éventuelle.

3 Mesure de sécurité visant à éviter que le grimpeur se blesse en cas de chute.

\section{Bibliographie}

Alpine Adventures Inc. (2004), Encouraging Environmental Stewardship, 7 p., [http://www. alpineadven.com/ourvalues/steward.html].

Cole, David N., Alan E. Watson, Troy E. Hall, et al. (1997), High-use Destinations in Wilderness: Social and Biophysical Impacts, Visitor Responses, and Management Options, Research Paper INT-RP-496, Ogden, UT, US Department of Agricultural Forest Service, Intermountain Research Station, $30 \mathrm{p}$.

Dubuc, Patrick, Alex Gerrits, et Raymond Tardif (n.d.), Le bloc du Mt. Wright: Stonebleau, 23 p., [http://www.drtopo.com/quebec/stone bleau.html].

Elle, Dana (2000), Minimizing Impact and Random Acts of Kindness for the Environment, Idaho State University, 7 p., [http://www.isu.edu/ outdoor/minimal.htm].

Jackson, Siobhan, et Doug Leavers (2000), "Determining a Capacity for Recreation and Monitoring Effects from Commercial Recreation Operations on Crown Land in BC », Recreation Series Occasional Paper, $n^{\circ} 1$.

Leung, Yu-Fai, et Jeffrey L. Marion (1996), «Trail Degradation as Influenced by Environmental Factors: A State-of-Knowledge Review ", Journal of Soil and Water Conservation, mars-avril, p. 130-136.

Leung, Yu-Fai, et Jeffrey L. Marion (1999), « Chapter $\mathrm{V}$. The Influence of Sampling Interval on the Accuracy of Trail Impact Assessment ", Landscape and Urban Planning, vol. 43, $\mathrm{n}^{\circ} 4$, p. 167-179.

Leung, Yu-Fai, et Jeffrey L. Marion (2000), «Recreation Impacts and Management in Wilderness: A State-of-Knowledge Review ", US Department of Agriculture Forest Service, Proceeding RMRS-P-15, vol. 5, p. 23-35.

Marion, Jeffrey L. (1995), «Capabilities and Management Utility of Recreation Impact Monitoring Programs ", Environmental Management, vol. 19, n 5, p. 763-771.

Parcs Québec (2005), Programme de suivi de l'intégrité écologique (PSIE), document de travail, Comité sur l'intégrité écologique de Parcs Québec, 55 p.

Watson, Alan, et Doug Cole (1992), Ideas for Limits of Acceptable Change Process, Book Two: LAC Indicators an Evaluation of Progress and List of Proposed Indicators, Washington DC, US Department of Agriculture Forest Service. 